\title{
A Distribution of Information Granularity to Deal With Inconsistency in Multi-Criteria and Heterogeneous Group Decision Making
}

\author{
F.J. Cabrerizo \\ University of Granada \\ cabrerizo@decsai.ugr.es
}

\author{
I.J. Pérez \\ University of Cádiz \\ ignaciojavier.perez@uca.es
}

\author{
J.A. Morente-Molinera \\ University of Granada \\ jamoren@decsai.ugr.es
}

\author{
E. Herrera-Viedma \\ University of Granada \\ viedma@decsai.ugr.es
}

\begin{abstract}
A distribution of information granularity has been implemented in several group decision making approaches to improve the individual consistency in the recent past. However, these approaches cannot be applied for solving decision processes carried out in multi-criteria and heterogeneous contexts. To overcome this shortcoming, we develop a new group decision making model in this study. First, considering fuzzy preference relations, the information granularity is built by means of distributing the flexibility degrees, which are required to improve the consistency, to the individuals. Second, it can handle group decision making processes in which several criteria, having distinct importance weights, are considered. Third, it considers that the preferences communicated by a decision maker have a not identical importance weight for every criterion. To illustrate the proposed group decision making model, a study is carried out via a numerical example. The observations show that this group decision making model can produce consistent decisions.
\end{abstract}

\section{Introduction}

Let $D M=\left\{d m^{1}, \ldots, d m^{m}\right\}$ be a finite group of decision makers and let $A=\left\{a_{1}, \ldots, a_{n}\right\}$ be a finite collection of alternatives. In group decision making processes carried out in fuzzy contexts, the final objective is to assign a preference degree in $[0,1]$ to each alternative in line with the preferences communicated by the decision makers $[1,2]$. To do so, it is commonly assumed that every decision maker, $d m^{h}$, communicates her or his preferences over pairs of alternatives, $a_{i}$ and $a_{j}$, via a fuzzy preference relation, $P R^{h}$, which is defined as a fuzzy set over all pairs of the Cartesian product $A \times A$, so that its membership function, $\mu$ : $A \times A \rightarrow[0,1]$, associates to every pair of alternatives $\left(a_{i}, a_{j}\right)$ the strength or intensity of preference [3, 4].

The advantage of using fuzzy preference relations is that of focusing only on two alternatives once at a time [5]. It decreases hesitation and uncertainty in comparison to assign preference degrees to all alternatives included in the collection in an only one step, which requires that a decision maker has to be capable of comparing every alternative against all the others as a whole. And this is commonly a challenging job. However, the use of fuzzy preference relations can lead to inconsistent preferences [6]. To clarify it, consider a decision maker expressing her or his preferences over this collection of three alternatives $\left\{a_{1}, a_{2}, a_{3}\right\}$. Let us suppose the decision maker's preferences are: $a_{2}$ is better than $a_{1}, a_{1}$ is better than $a_{3}$, and $a_{3}$ is better than $a_{2}$. Is this decision maker consistent? Obviously not, whether $a_{2}$ is better than $a_{1}$ and $a_{1}$ is better than $a_{3}$, then $a_{2}$ must be better than $a_{3}$.

Consistency has been considered as a rationality measure [7], which is obviously a fuzzy concept as a certain amount of individual preferences may inherently be judged as more inconsistent than others. Therefore, to each decision maker, one can assign a consistency degree in $[0,1]$, where 1 means absolute consistency and 0 means absolute inconsistency. Several properties, to be fulfilled by a fuzzy preference relation, have been suggested to make a rational choice [6]. They may be utilized to measure the consistency degree assigned to a decision maker. In addition, based on them, different procedures have been developed to deal with inconsistency in fuzzy preference relations [8].

Recently, some approaches based on a distribution of information granularity, which increases the consistency assigned to each decision maker before assigning the preference degree to each alternative, have been developed to support group decision making processes $[9,10,11]$. These approaches based on a distribution of information granularity assume the decision makers judge their preferences between pairs of alternatives as a whole, but, to model group decision making processes in a more realistic way, a number of criteria must be considered by the decision makers when comparing two alternatives [12, 13, 14]. For example, the LibQUAL+ methodology makes use of different 
criteria, as for example "quite space for individual activities" or "print and/or electronic journal collections I require for my work", to evaluate the library service quality [15]. Furthermore, due to the background and knowledge of each decision maker, the group decision making approaches should consider that the preferences communicated by a decision maker have a different importance depending on the criterion. Continuing with the above example, the researcher's preference on "print and/or electronic journal collections I require for my work" should be more important than her or his preference on "quite space for individual activities". Based on a similar reasoning, the importance of each criterion should be different when the criteria are considered to obtain the final preference degree associated with each alternative $[16,17]$. As a result, because the framework is heterogeneous [18], there must be an allowance for such differences in importance.

In this study, taking into account these considerations, we develop a new group decision making model dealing with inconsistency in decision processes carried out in multi-criteria and heterogeneous settings. To handle the inconsistency, a distribution of information granularity is injected into the fuzzy preference relations to allow a flexibility degree that will be used to decrease the inconsistency associated with the fuzzy preference relations. Furthermore, and unlike the existing models based on a distribution of information granularity, this new model has the ability to support group decision making processes where more than one criterion is kept in mind to evaluate the alternatives and in which the heterogeneity is considered from two points of view: (i) the preferences communicated by a decision maker have a distinct weight of importance for every criterion, and (ii) the criteria considered to evaluate the alternatives have different importance weights.

This study is organized as follows. Section 2 recalls the necessary background theory. The group decision making model proposed in this study is formally defined in Section 3. Section 4 conducts an experimental study and shows its results. The performance and characteristics of the proposed model are discussed in Section 5. Conclusions and future research directions are covered in Section 6.

\section{Background}

This section gives the necessary background information for the model that is developed in the next section. Concretely, it covers the fuzzy majority concept, and its modelling via fuzzy quantifiers, and the description of the particle swarm optimization (PSO).

\subsection{Fuzzy majority}

A number of individuals representing a threshold has usually been adopted to characterize the concept of majority. However, when dealing with group decision making problems, it is considered more appropriate to adopt a soft concept of majority, called fuzzy majority, that is characterized by a fuzzy linguistic quantifier [19].

The quantity of objects satisfying a particular predicate may be represented by a quantifier. For instance, classical logic only considers two quantifiers: "for all", which is associated with the "and" connective, and "there exists", which is associated with the "or" connective. Nevertheless, the human discourse offers a greater number of quantifiers, for instance, "about seven", "at least half", "most", "a few", etc. With the aim of bridging the gap between human discourse and formal systems and, subsequently, to offer a knowledge representation tool more flexible, Zadeh introduced the fuzzy quantifiers [19], which were employed by Kacprzyk to represent the fuzzy majority in group decision making [2].

Fuzzy subsets have been adopted to capture the semantics related to a fuzzy quantifier. In particular, fuzzy quantifiers can be categorized into two types:

- Absolute quantifiers. They describe quantities that are absolute in nature ("more than eight" or "about three") and are characterized by fuzzy subsets of $\mathbb{R}^{+}$. Here, a fuzzy subset $Q$ represents an absolute quantifier such that for any $r \in \mathbb{R}^{+}$, the degree in which the quantity $r$ is compatible with the quantifier symbolized by $Q$ is determined by $Q(r)$, which represents the membership degree of $r$ in $Q$. In particular, an absolute quantifier, $Q: \mathbb{R}^{+} \rightarrow[0,1]$, satisfies the following two conditions: $\exists k$ such that $Q(k)=1$ and $Q(0)=0$.

- Relative quantifiers. They describe a statement of proportion type ("at least half" or "most") and are characterized by fuzzy subsets of $[0,1]$. Here, a fuzzy subset $Q$ represents a relative quantifier such that for any $r \in[0,1]$, the degree in which the proportion $r$ is compatible with the quantifier symbolized by $Q$ is determined by $Q(r)$, which represents the membership degree of $r$ in $Q$. In particular, a relative quantifier, $Q:[0,1] \rightarrow[0,1]$, satisfies the following two conditions: $\exists r \in[0,1]$ such that $Q(r)=1$ and $Q(0)=0$.

In [20], two types of relative quantifiers were recognized by Yager: regular decreasing monotone quantifiers (for instance, "at most $\gamma$ " or "few") and regular increasing monotone quantifiers (for instance, 


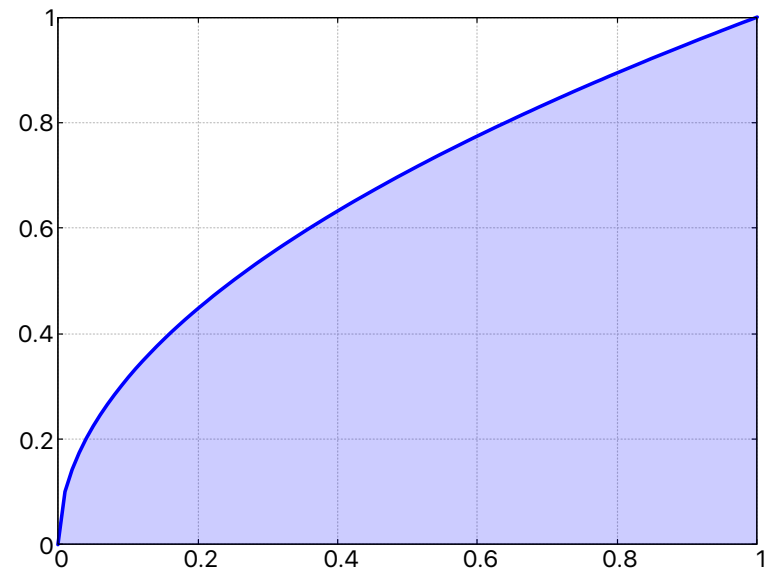

Figure 1. Relative fuzzy quantifier "most".

"at least $\gamma$ ", "many", "most", or "all"). The latter satisfies $\forall x, y$ if $x>y$ then $Q(x)>Q(y)$.

By way of illustration, we can consider the following regular increasing monotone function that characterizes the fuzzy quantifier "most" (see Fig. 1):

$$
Q(r)=r^{1 / 2}
$$

\subsection{Particle swarm optimization}

This optimization algorithm, attributed to Eberhart and Kennedy [21], had as its initial purpose the imitation of social behaviour by trying to represent the movement of organisms in a fish school or a bird flock [22]. Given a problem, this algorithm finds the optimal solution in an iterative way by trying to make better candidate solutions concerning a particular quality measure. Considering a population, also known as a swarm, of candidate solutions, also known as particles, this algorithm optimizes the problem by moving these candidate solutions in all directions of the candidate solutions space in line with simple mathematical formulae. The movement of each particle is guided towards its best known location as well as it is influenced by the best known location in the space of candidate solutions that represents the best position discovered by the remaining particles. The objective is to guide the population of candidate solutions towards the best solution through evaluation of several generations of them.

Formally, let $m$ and $n$ be the swarm size and the search space size, respectively. Let $\mathbf{v}_{j} \in \mathbb{R}^{n}$ and $\mathbf{x}_{j} \in \mathbb{R}^{n}$ be the velocity vector and the position vector in the $n$-dimensional search space, respectively, that are associated with the particle $j \in\{1, \ldots, m\}$. Let $g: \mathbb{R}^{n} \rightarrow \mathbb{R}$ a cost function, also called fitness function, that has to be optimized (we suppose in this case it must be maximized). This fitness function receives the vector of real numbers $\mathbf{x}_{j}$ as argument and generates an output in the form of real number indicating the value of the objective function associated with the particle $j \in\{1, \ldots, m\}$. The objective is to locate a particle $j$ for which $g\left(\mathbf{x}_{j}\right) \geq g\left(\mathbf{x}_{k}\right)$ for all $k \in\{1, \ldots, m\}$ and $j \neq k$. It signifies the particle $j$ would be a global maximum. Let $\mathbf{p b}_{j}$ be the best location the particle $j$ has ever reached and let gb be the best location of the swarm obtained up to this point. Let $x_{\max }$ and $x_{\min }$ be the upper and lower limits of the search space, respectively. Let $c_{1}$ be the cognitive learning factor influencing the step size taken by the particle towards its best location, and let $c_{2}$ be the social learning factor representing the step size taken by the particle towards the global optimal position in the swarm. Let $\omega$ the inertia weight balancing the global search, exploration, and the local search, exploitation [23] (a bigger value of $\omega$ is geared towards exploration whereas a smaller value of $\omega$ is tended to exploitation). Some advantages of the PSO include its ease of executing through programming, its fast convergence rate and its low number of parameters to be adjusted [24]. Algorithm 1 describes the pseudocode of a basic PSO algorithm.

\section{A model based on a distribution of information granularity to deal with inconsistency}

This section is devoted to introduce a new model based on a distribution of information granularity to address inconsistency in group decision making processes carried out in multi-criteria and heterogeneous settings.

This kind of decision process is formalized as follows. Let $C=\left\{c^{1}, \ldots, c^{q}\right\}$ be a set of $q$ criteria. Let $A=\left\{a_{1}, \ldots, a_{n}\right\}$ be a collection of $n$ alternatives. Let $D M=\left\{d m^{1}, \ldots, d m^{m}\right\}$ be a group of $m$ decision makers. Let $\alpha^{k}$ be the weight of importance associated with each criterion $c^{k}$. Let $\beta^{h k}$ be the weight of importance associated with the preferences provided by the decision maker $d m^{h}$ when he or she considers the criterion $c^{k}$. The decision makers communicate their preferences over the alternatives, keeping in mind the criteria, to arrive at a global preference degree assigned to every alternative [25]. The objective is to select the alternative, or the alternatives, whose global preference degree is higher.

As mentioned in Section 1, we suppose the decision makers communicate their preferences with the aid of fuzzy preference relations. Therefore, every decision maker $d m^{h}$ provides a fuzzy preference relation $P R^{h k}$ for every criterion, $c^{k}$. When all the decision makers 


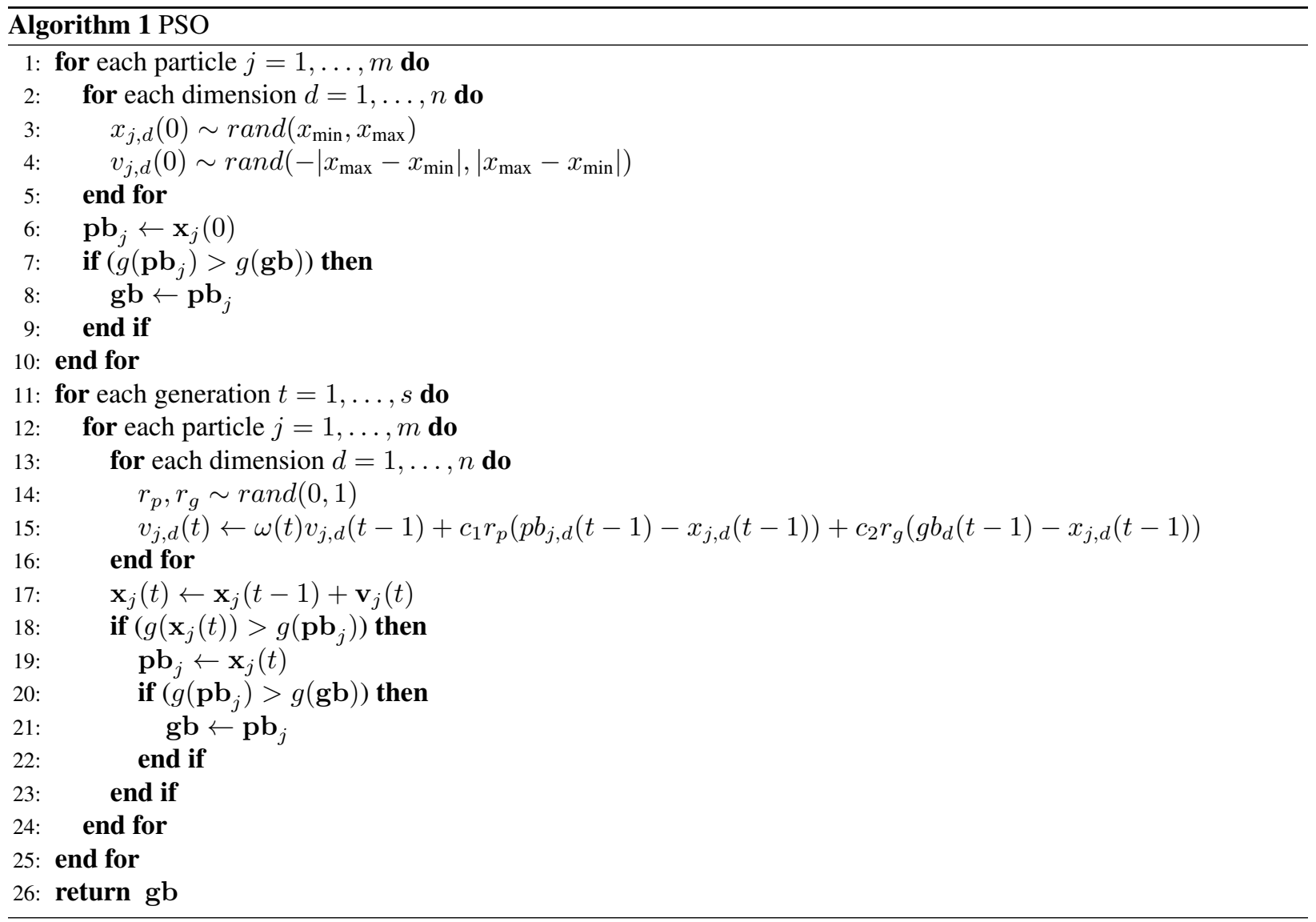

have communicated the fuzzy preference relations required, the proposed model, which is structured into two steps, is applied. First, a distribution of information granularity is injected into every fuzzy preference relation to deal with the inconsistency. Second, a selection process is executed to select the alternative, or alternatives, having the higher global preference degree. In the next subsections, we elaborate on them.

\subsection{Distribution of information granularity to deal with the inconsistency}

Whether a preference representation format like fuzzy preference relations or another based on pairwise comparisons is used, it is important to deal with the inconsistency for the purpose of avoiding misleading decisions [7, 8]. Of course, it requires the decision maker changes her or his mind.

To decrease the inconsistency related to the fuzzy preference relation expressed by a decision maker, he or she must change his or her original preferences, which implies a certain flexibility degree in his or her initial preferences. We can deal with this by managing every element of the fuzzy preference relation as an information granule [26], which acts here as a substitute for an exact numeric value. This leads to the granular format, $\mathbf{G}(P R)$, of the fuzzy preference relation, $P R$.

The target is to put the information granularity [27] to work in order to decrease the inconsistency (in other words, to increase the consistency) related to a fuzzy preference relation. In brief, we handle the level of information granularity, which emerges here as a helpful computation and conceptual tool applied to minimize the inconsistency related to a fuzzy preference relation, as a synonym for the level of flexibility.

Following the ideas introduced in $[9,10,11]$, we assume intervals as specific granular formalism, $\mathbf{G}(\cdot)$. Consequently, $\mathbf{G}(P R)=\mathbf{I}(P R)$, representing $\mathbf{I}(\cdot)$ a family of intervals, and, in this instance, the level of information granularity may be naturally equated to the length of the intervals. Then, the essence of the consistency can be captured by an optimization criterion that is optimized by means of the flexibility provided by the length of the intervals. This is to say, whether the decision makers feel equally confident when choosing any fuzzy preference relation whose elements assume values located inside the limits of the intervals, it can be used to decrease the inconsistency related to 
the fuzzy preference relation. Consequently, the next optimization criterion can be established to effectuate this improvement of consistency:

$$
O=\frac{1}{m} \sum_{h=1}^{m} c d^{h}
$$

where $c d^{h}$ denotes the consistency associated with the preferences expressed by the decision maker $d m^{h}$, which is calculated as follows:

$$
c d^{h}=\frac{1}{\sum_{k=1}^{q} \beta^{h k}} \sum_{k=1}^{q} \beta^{h k} \cdot c d^{h k}
$$

where $c d^{h k}$ denotes the consistency related to the fuzzy preference relation expressed by the decision maker $d m^{h}$ when he or she considers the criterion $c^{k}$. The model developed in [28] is applied to compute this consistency degree, although other methodologies could be also used [8].

In summary, the optimization task of the fuzzy preference relations coming from the space of interval fuzzy preference relations reads as follows:

$$
\max _{P R^{1}, \ldots, P R^{m} \in \mathbf{I}(P R)} O
$$

To maximize the optimization criterion $O$ is not easy, but as shown in $[9,10,11]$, where comparable optimization tasks were performed, the PSO is a good choice to do it and, therefore, this algorithm is also used in this study. Next, we elaborate on the structure of the particle and the fitness function employed in this particular optimization task.

3.1.1. Particle Considering the framework assumed in this study, a particle is characterized by a vector whose elements assume numeric values located in $[0,1]$, which corresponds to the search space. Then, if there are $m$ decision makers, $n$ alternatives and $q$ criteria, a particle is composed of $n \cdot(n-1) \cdot m \cdot q$ elements.

Considering a level of information granularity $\gamma \in[0,2]$ and the preference degree $p r_{i j}^{h k}$ of the fuzzy preference relation $P R^{h k}$ communicated by the decision maker $d m^{h}$ over the alternatives $a_{i}$ and $a_{j}$ when he or she considers the criterion $c^{k}$, the possible values assumed by this preference degree are located within the boundaries of the following interval:

$$
[a, b]=\left[\max \left(0, p r_{i j}^{h k}-\gamma / 2\right), \min \left(p r_{i j}^{h k}+\gamma / 2,1\right)\right]
$$

Then, assuming an element $x$ of the particle, it is transformed linearly by means of:

$$
z=a+(b-a) \cdot x
$$

To clarify it, let us suppose $p r_{i j}^{h k}=0.4, x=0.7$ and $\gamma=0.6$. According to these values, the corresponding interval related to the preference degree $p r_{i j}^{h k}$ that is obtained using (5) is $[0.1,0.7]$. Then, the new value related to $p r_{i j}^{h k}$ is 0.52 according to (6).

3.1.2. Fitness function Because the PSO is applied to maximize the consistency of the fuzzy preference relations communicated by the decision makers, the fitness function $g$, which is adopted to measure the quality of a given particle, is determined in the following way:

$$
g=O
$$

\subsection{Selection process}

This second step consists in assigning a global preference degree in $[0,1]$ to each alternative, in line with the fuzzy preference relations communicated by the decision makers, to select the alternative, or alternatives, whose global preference degree is higher [28]. To do this, we split it into two sub-steps, aggregation and exploitation, that are both based on the concept of fuzzy majority.

3.2.1. Aggregation It generates a collective fuzzy preference relation, $P R^{c}$, summarizing the preferences communicated by the decision makers. According to the setting assumed, this sub-step must consider that the preferences expressed by a decision maker have associated a weight of importance for each criterion and that each criterion also has a weight of importance. Therefore, an IOWA operator should be used [29].

Definition 1 An IOWA operator of dimension $n$ is a mapping $\Phi: \mathbb{R}^{n} \times \mathbb{R}^{n} \rightarrow \mathbb{R}$ having an associated weighting vector $w=\left(w_{1}, \ldots, w_{n}\right)$, such that $\sum_{i=1}^{n} w_{i}=1$ and $w_{i} \in[0,1]$, and with:

$$
\Phi\left(\left\langle u_{1}, a_{1}\right\rangle, \ldots,\left\langle u_{n}, a_{n}\right\rangle\right)=\sum_{j=1}^{n} w_{j} \cdot b_{j}
$$

where $b_{j}$ is the $a_{i}$ element (also called argument variable) of the pair $\left\langle u_{i}, a_{i}\right\rangle$ having the $j$-th largest $u_{i}$ value (also called order inducing variable).

The IOWA operator puts the fuzzy majority concept into effect thanks to a fuzzy quantifier [19] indicating 
the proportion of satisfied criteria "necessary for a good solution" [20]. Notably, this is done by using the fuzzy quantifier to compute the elements of the weighting vector $w$. In [20], Yager proposed a procedure to do this in which, once the values to be combined have been ordered, the following expression is used to compute the weighting vector related to the IOWA operator using a fuzzy quantifier $Q$ :

$$
w_{i}=Q\left(\frac{\sum_{j=1}^{i} b_{j}}{\sum_{j=1}^{n} u_{j}}\right)-Q\left(\frac{\sum_{j=1}^{i-1} b_{j}}{\sum_{j=1}^{n} u_{j}}\right)
$$

where $b_{j}$ is the $j$-th largest $u_{i}$ value.

This methodology for including weights of importance associates a weight equal to 0 with the decision makers having a weight of importance equal to 0 . As depicted in Fig. 1, the fuzzy quantifier "most" defined in (1) is a strictly increasing function. On one hand, it guarantees that all the preferences provided by the decision makers whose weight of importance is nonzero make a contribution to the aggregated value. On the other, it associates a high weighting value with the preferences that have a high weight of importance. Therefore, in this study, we use this fuzzy quantifier to calculate the weights associated with the IOWA operator used. When the weights associated with an IOWA operator are estimated by means of a fuzzy quantifier $Q$, it is represented by $\Phi_{Q}$.

The methodology applied to compute the collective fuzzy preference relation, which has to take into account all the fuzzy preference relations communicated by all the decision makers, is as follows:

- Using the IOWA operator, a collective fuzzy preference relation, $P R^{c k}$, is computed for every criterion $c^{k}$ as follows:

$$
p r_{i j}^{c k}=\Phi_{Q}\left(\left\langle\beta^{1 k}, p r_{i j}^{1 k}\right\rangle, \ldots,\left\langle\beta^{m k}, p r_{i j}^{m k}\right\rangle\right)
$$

Here, the order inducing variable is the weight of importance related to the preferences provided by a decision maker over the criterion $c^{k}$.

- Using again the IOWA operator, we compute the final collective fuzzy preference relation $P R^{c}$ in the following way:

$$
p r_{i j}^{c}=\Phi_{Q}\left(\left\langle\alpha^{1}, p r_{i j}^{c 1}\right\rangle, \ldots,\left\langle\alpha^{k}, p r_{i j}^{c k}\right\rangle\right)
$$

In this case, the weight of importance related to each criterion $c^{k}$ is used as order inducing variable.
3.2.2. Exploitation In line with the information contained in the collective fuzzy preference relation $P R^{c}$, this sub-step assigns a global preference degree in $[0,1]$ to each alternative by means of a pair of choice degrees of alternatives. They are the quantifier-guided dominance degree, $Q G D D_{i}$, that measures the degree in which the alternative $a_{i}$ dominates the others in a fuzzy majority sense, and the quantifier-guided non-dominance degree, $Q G N D D_{i}$, that measures the degree in which the alternative $a_{i}$ is not dominated by a fuzzy majority of the others [28, 30]. Both choice degrees are founded on the fuzzy majority concept and the OWA operator [31].

Definition 2 An OWA operator of dimension $n$ is a mapping $\phi: \mathbb{R}^{n} \rightarrow \mathbb{R}$ having an associated weighting vector $w=\left(w_{1}, \ldots, w_{n}\right)$, such that $\sum_{i=1}^{n} w_{i}=1$ and $w_{i} \in[0,1]$, and with:

$$
\phi\left(a_{1}, \ldots, a_{n}\right)=\sum_{j=1}^{n} w_{j} \cdot b_{j}
$$

where $b_{j}$ is the $j$-th largest element in $a_{1}, \ldots, a_{n}$.

In the case of the OWA operator, an approach also based on fuzzy quantifiers may be used to obtain the weighting vector $w$. In particular, Yager proposed to use the following expression:

$$
w_{i}=Q\left(\frac{i}{n}\right)-Q\left(\frac{i-1}{n}\right)
$$

When the weights associated with an OWA operator are estimated by means of a fuzzy quantifier $Q$, it is represented by $\phi_{Q}$.

The methodology applied to select the alternative, or alternatives, having the highest total preference degree is as follows:

- For each alternative, $a_{i}$, we obtain its quantifier-guided dominance degree:

$$
\begin{aligned}
Q G D D_{i}= & \phi_{Q}\left(p r_{i 1}^{c}, \ldots, p r_{i(i-1)}^{c}\right. \\
& \left.p r_{i(i+1)}^{c}, \ldots, p r_{i n}^{c}\right)
\end{aligned}
$$

- For each alternative, $a_{i}$, we obtain its quantifier-guided non-dominance degree:

$$
\begin{aligned}
Q G N D D_{i}= & \phi_{Q}\left(1-p r_{1 i}^{s}, \ldots, 1-p r_{(i-1) i}^{s}\right. \\
& \left.1-p r_{(i+1) i}^{s}, \ldots, 1-p r_{n i}^{s}\right)
\end{aligned}
$$

where $p r_{j i}^{s}=\max \left(p r_{j i}^{c}-p r_{i j}^{c}, 0\right)$ establishes the degree in which the alternative $a_{j}$ dominates completely to the alternative $a_{i}$. 
- For each choice degree, a collection of alternatives is determined as follows:

$$
\begin{gathered}
A^{D D}=\begin{array}{l}
\left\{a_{i} \in A \mid Q G D D_{i}=\right. \\
\left.\sup _{a_{j} \in A} Q G D D_{j}\right\}
\end{array} \\
A^{N D D=} \begin{array}{l}
\left\{a_{i} \in A \mid Q G N D D_{i}=\right. \\
\left.\sup _{a_{j} \in A} Q G N D D_{j}\right\}
\end{array}=
\end{gathered}
$$

- The intersection of both collections generates a new collection of alternatives:

$$
A^{Q G}=A^{D D} \cap A^{N D D}
$$

If $A^{Q G} \neq \emptyset$, we select the alternative, or alternatives, of this collection. If not, continue.

- If $\# A^{D D}=1$, we select the alternative of this collection. If not, we select the alternative, or alternatives, of this collection having the highest quantified-guided non-dominance degree.

\section{Experimental study}

Suppose that three decision makers $D M=$ $\left\{d m^{1}, d m^{2}, d m^{3}\right\}$ evaluate four alternatives, $A=$ $\left\{a_{1}, a_{2}, a_{3}, a_{4}\right\}$ considering three criteria, $C=$ $\left\{c^{1}, c^{2}, c^{3}\right\}$. In addition, suppose that the weights of importance related to the criteria are $\alpha^{1}=0.5, \alpha^{2}=0.3$ and $\alpha^{3}=0.2$, and that the weights of importance related to the preferences provided by the decision makers over each criterion are $\beta^{11}=0.5, \beta^{12}=0.2, \beta^{13}=0.3$, $\beta^{21}=0.2, \beta^{22}=0.3, \beta^{23}=0.5, \beta^{31}=0.3, \beta^{32}=0.5$ and $\beta^{33}=0.2$.

Initially, the decision makers provide the following fuzzy preference relations:

$$
\begin{aligned}
P R^{11} & =\left(\begin{array}{cccc}
- & 0.30 & 0.50 & 0.50 \\
0.50 & - & 0.70 & 0.70 \\
0.50 & 0.30 & - & 0.70 \\
0.50 & 0.30 & 0.30 & -
\end{array}\right) \\
P R^{12} & =\left(\begin{array}{cccc}
- & 0.30 & 0.90 & 0.90 \\
0.70 & - & 0.10 & 0.10 \\
0.10 & 0.90 & - & 0.70 \\
0.10 & 0.90 & 0.30 & -
\end{array}\right) \\
P R^{13} & =\left(\begin{array}{cccc}
- & 0.30 & 0.30 & 0.70 \\
0.90 & - & 0.30 & 0.30 \\
0.90 & 0.70 & - & 0.30 \\
0.50 & 0.70 & 0.90 & -
\end{array}\right) \\
P R^{21} & =\left(\begin{array}{cccc}
- & 0.50 & 0.30 & 0.90 \\
0.50 & - & 0.90 & 0.90 \\
0.70 & 0.10 & - & 0.50 \\
0.10 & 0.10 & 0.50 & -
\end{array}\right)
\end{aligned}
$$

$$
\begin{aligned}
P R^{22} & =\left(\begin{array}{cccc}
- & 0.70 & 0.70 & 0.70 \\
0.10 & - & 0.90 & 0.30 \\
0.50 & 0.30 & - & 0.90 \\
0.50 & 0.50 & 0.30 & -
\end{array}\right) \\
P R^{23} & =\left(\begin{array}{cccc}
- & 0.50 & 0.50 & 0.30 \\
0.50 & - & 0.50 & 0.70 \\
0.50 & 0.50 & - & 0.50 \\
0.90 & 0.10 & 0.50 & -
\end{array}\right) \\
P R^{31} & =\left(\begin{array}{cccc}
- & 0.10 & 0.70 & 0.30 \\
0.70 & - & 0.50 & 0.30 \\
0.30 & 0.50 & - & 0.50 \\
0.70 & 0.70 & 0.50 & -
\end{array}\right) \\
P R^{32} & =\left(\begin{array}{cccc}
- & 0.90 & 0.90 & 0.70 \\
0.30 & - & 0.70 & 0.50 \\
0.30 & 0.10 & - & 0.70 \\
0.30 & 0.50 & 0.30 & -
\end{array}\right) \\
P R^{33} & =\left(\begin{array}{cccc}
- & 0.30 & 0.30 & 0.70 \\
0.50 & - & 0.50 & 0.70 \\
0.30 & 0.50 & - & 0.50 \\
0.30 & 0.70 & 0.90 & -
\end{array}\right)
\end{aligned}
$$

Before showing the results generated by the first step of the proposed method, we detail the values assigned to the parameters of the PSO: $c_{1}$ and $c_{2}$ were set to 2; the number of generations run was 500; and the swarm consisted of 200 particles. These values were chosen as a result of an intense experimentation. In addition, the level of information granularity $\gamma$ was set to 0.2 .

The PSO returns a value of 0.904 for the optimization criterion $O$ and the following adjusted fuzzy preference relations:

$$
\begin{aligned}
P R^{11} & =\left(\begin{array}{cccc}
- & 0.40 & 0.50 & 0.60 \\
0.60 & - & 0.60 & 0.70 \\
0.50 & 0.40 & - & 0.80 \\
0.40 & 0.30 & 0.40 & -
\end{array}\right) \\
P R^{12} & =\left(\begin{array}{cccc}
- & 0.40 & 0.80 & 0.80 \\
0.60 & - & 0.20 & 0.20 \\
0.20 & 0.80 & - & 0.60 \\
0.20 & 0.80 & 0.40 & -
\end{array}\right) \\
P R^{13} & =\left(\begin{array}{cccc}
- & 0.40 & 0.40 & 0.60 \\
0.80 & - & 0.40 & 0.40 \\
0.80 & 0.60 & - & 0.40 \\
0.60 & 0.60 & 0.80 & -
\end{array}\right) \\
P R^{21} & =\left(\begin{array}{cccc}
- & 0.40 & 0.40 & 1.00 \\
0.60 & - & 0.80 & 0.97 \\
0.60 & 0.20 & - & 0.60 \\
0.20 & 0.00 & 0.40 & -
\end{array}\right)
\end{aligned}
$$




$$
\begin{aligned}
P R^{22} & =\left(\begin{array}{cccc}
- & 0.60 & 0.60 & 0.73 \\
0.20 & - & 0.80 & 0.40 \\
0.40 & 0.40 & - & 0.80 \\
0.40 & 0.40 & 0.40 & -
\end{array}\right) \\
P R^{23} & =\left(\begin{array}{cccc}
- & 0.40 & 0.42 & 0.40 \\
0.60 & - & 0.60 & 0.60 \\
0.60 & 0.40 & - & 0.49 \\
0.80 & 0.20 & 0.51 & -
\end{array}\right) \\
P R^{31} & =\left(\begin{array}{cccc}
- & 0.20 & 0.60 & 0.33 \\
0.60 & - & 0.60 & 0.40 \\
0.40 & 0.40 & - & 0.40 \\
0.60 & 0.60 & 0.60 & -
\end{array}\right) \\
P R^{32} & =\left(\begin{array}{cccc}
- & 0.80 & 0.80 & 0.80 \\
0.40 & - & 0.60 & 0.60 \\
0.20 & 0.20 & - & 0.60 \\
0.20 & 0.40 & 0.40 & -
\end{array}\right) \\
P R^{33} & =\left(\begin{array}{cccc}
- & 0.40 & 0.40 & 0.60 \\
0.40 & - & 0.60 & 0.60 \\
0.20 & 0.40 & - & 0.47 \\
0.40 & 0.60 & 1.00 & -
\end{array}\right)
\end{aligned}
$$

Once the consistency has been increased, the selection process is applied to get the solution. Using (10) and the fuzzy quantifier "most" defined in (1) to generate the weighting vector of the IOWA operator according to (9), we obtain the following collective fuzzy preference relations for each criterion:

$$
\begin{aligned}
P R^{c 1} & =\left(\begin{array}{cccc}
- & 0.36 & 0.51 & 0.59 \\
0.60 & - & 0.62 & 0.67 \\
0.49 & 0.38 & - & 0.70 \\
0.42 & 0.32 & 0.44 & -
\end{array}\right) \\
P R^{c 2} & =\left(\begin{array}{cccc}
- & 0.72 & 0.76 & 0.79 \\
0.38 & - & 0.59 & 0.52 \\
0.24 & 0.30 & - & 0.70 \\
0.42 & 0.32 & 0.44 & -
\end{array}\right) \\
P R^{c 3} & =\left(\begin{array}{cccc}
- & 0.40 & 0.41 & 0.46 \\
0.62 & - & 0.56 & 0.56 \\
0.59 & 0.44 & - & 0.47 \\
0.72 & 0.32 & 0.62 & -
\end{array}\right)
\end{aligned}
$$

The final collective preference relation $P R^{c}$ is computed by combining $P R^{c 1}, P R^{c 2}$ and $P R^{c 3}$. To do so, (11) is used with the fuzzy quantifier "most" defined in (1) to generate the weighting vector according to (9):

$$
P R^{c}=\left(\begin{array}{cccc}
- & 0.43 & 0.55 & 0.61 \\
0.56 & - & 0.61 & 0.63 \\
0.45 & 0.37 & - & 0.68 \\
0.45 & 0.32 & 0.46 & -
\end{array}\right)
$$

Finally, using (14) and (15), with the fuzzy quantifier "most" defined in (1) to generate the weighting vector of the OWA operator according to (13), we obtain the following quantifier-guided dominance degrees and the quantifier-guided non-dominance degrees, respectively:

$$
\begin{array}{ll}
Q G D D_{1}=0.56 & Q G D D_{2}=0.61 \\
Q G D D_{3}=0.57 & Q G D D_{4}=0.43 \\
Q G N D D_{1}=0.97 & Q G N D D_{2}=1.00 \\
Q G N D D_{3}=0.93 & \\
Q G N D D_{4}=0.80
\end{array}
$$

According to these values, $A^{D D}=\left\{a_{2}\right\}, A^{N D D}=$ $\left\{a_{2}\right\}$, and $A^{Q G}=\left\{a_{2}\right\}$. Hence, the alternative $a_{2}$ is selected as solution to the problem.

\section{Discussion}

We analyze both the performance and characteristics of our proposal from different points of view in this section.

First, a matter of interest is to analyze how the consistency is improved or deteriorated. We quantify the effect of the level of information granularity $\gamma$ by generating in a random manner a fuzzy preference relation, $P R$, that comes from its interval representation, $\mathbf{I}(P R)$. Then, we compute its consistency. We repeat it 500 times for every value of $\gamma$. Fig. 2, Fig. 3 and Fig. 4 display the plots of the consistency in contrast to every value of $\gamma$ for the three fuzzy preference relations communicated by the first decision making in the experimental study described in Section 4. These plots also depict the average values of the consistencies. On one hand, if we increase the values of $\gamma$, we also increase the probability of arriving at a higher consistency. It is logical because we have put a certain flexibility level in place that

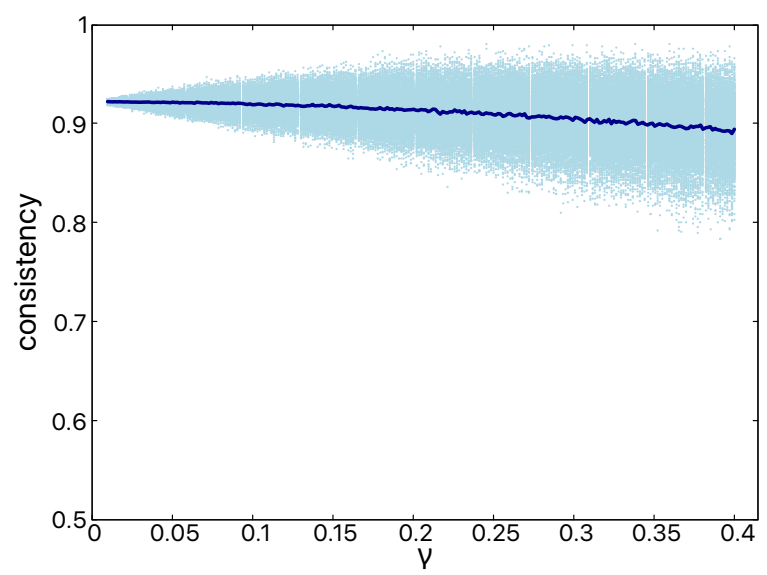

Figure 2. Consistency in contrast to $\gamma$ for $P R^{11}$. 


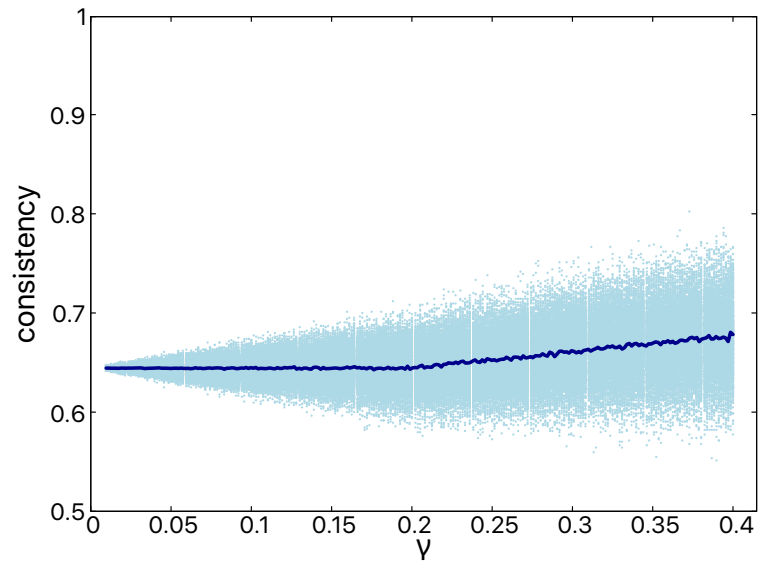

Figure 3. Consistency in contrast to $\gamma$ for $P R^{12}$.

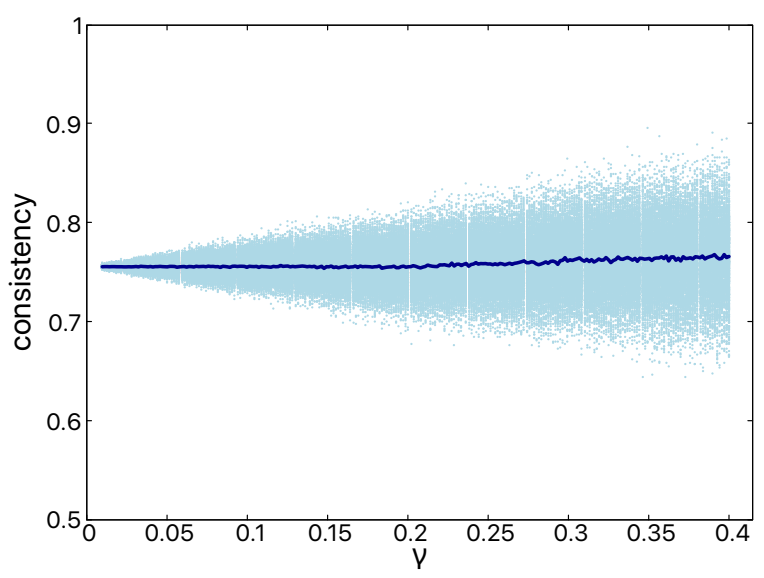

Figure 4. Consistency in contrast to $\gamma$ for $P R^{13}$.

we plan to exploit. On the other, we also increase the probability of producing a very inconsistent fuzzy preference relation. In any case, even though there exists a slight upward tendency when the consistency related to the fuzzy preference relation is initially low (see Fig. 3), and a slight downward tendency when the consistency is initially very high (see Fig. 2), the mean of the consistencies remains moderately stable respecting higher values of $\gamma$ (see Fig. 4).

Second, it becomes instructive to analyze the performance of the model against different values of $\gamma$. As we can observe in Table 1, the higher the value of $\gamma$, the higher the value of $O$. It means that a higher consistency is achieved, that is, higher values of $\gamma$ imply higher levels of flexibility injected into the fuzzy preference relations and, therefore, there is a higher probability of improving the consistency.
Table 1. Values of $O$ for selected values of $\gamma$.

\begin{tabular}{ccccc}
\hline & $\gamma=0.1$ & $\gamma=0.2$ & $\gamma=0.3$ & $\gamma=0.4$ \\
\hline$O$ & 0.868 & 0.904 & 0.938 & 0.967 \\
\hline
\end{tabular}

\section{Concluding remarks}

In this study, we have built a new group decision making model managing inconsistency in decision processes by virtue of a distribution of information granularity, which has been injected into the preferences communicated by the decision makers. Different from the developed methodologies based on a distribution of information granularity, this new model is able to deal with decision processes carried out in multi-criteria and heterogeneous contexts.

It has been observed that a higher level of information granularity allows to achieve a higher consistency, but it implies that the elements of the fuzzy preference relation could be associated with values very different from those communicated initially by the decision maker. As a result, the decision maker may not accept the new values obtained by the PSO. Consequently, an interesting future study could be to consider the similarity between the original preferences and the obtained by the PSO [32]. To do so, we can modify the optimization criterion in such a way that, in addition to the consistency, it considers the similarity between the original preferences and the calculated by the PSO. In addition, because only one experimental study has been carried out here, to better quantify the performance of the proposed model, more experimental studies varying the number of decision makers, alternatives and criteria, should be conducted.

\section{Acknowledgements}

This work has been supported by the Spanish State Research Agency through the project PID2019-103880RB-I00 / AEI / $10.13039 / 501100011033$.

\section{References}

[1] F. J. Cabrerizo, I. J. Pérez, J. A. Morente-Molinera, S. Alonso, and E. Herrera-Viedma, "An adaptive feedback mechanism for consensus reaching processes based on individuals' credibility," in Proceedings of the 52nd Hawaii International Conference on System Sciences, (Hawaii, USA), pp. 1678-1687, January 2019.

[2] J. Kacprzyk, "Group decision making with a fuzzy linguistic majority," Fuzzy Sets and Systems, vol. 18, no. 2, pp. 105-118, 1986.

[3] S. A. Orlovsky, "Decision-making with a fuzzy preference relation," Fuzzy Sets and Systems, vol. 1, no. 3 , pp. $155-167,1978$. 
[4] G. Zhang, Y. C. Dong, and Y. Xu, "Linear optimization modeling of consistency issues in group decision making based on fuzzy preference relations," Expert Systems with Applications, vol. 39, no. 3, pp. 2415-2420, 2012.

[5] I. Millet, "The effectiveness of alternative preference elicitation methods in the analytic hierarhcy process,' Journal of Multi-Criteria Decision Analysis, vol. 6, no. 1, pp. 41-51, 1997.

[6] E. Herrera-Viedma, F. Herrera, F. Chiclana, and M. Luque, "Some issues on consistency of fuzzy preference relations," European Journal of Operational Research, vol. 154, no. 1, pp. 98-109, 2004.

[7] V. Cutello and J. Montero, "Fuzzy rationality measures," Fuzzy Sets and Systems, vol. 62, no. 1, pp. 39-54, 1994.

[8] C.-C. Li, Y. C. Dong, Y. Xu, F. Chiclana, E. Herrera-Viedma, and F. Herrera, "An overview on managing additive consistency of reciprocal preference relations for consistency-driven decision making and fusion: Taxonomy and future directions," Information Fusion, vol. 52, pp. 143-156, 2019.

[9] F. J. Cabrerizo, R. Ureña, W. Pedrycz, and E. Herrera-Viedma, "Building consensus in group decision making with an allocation of information granularity," Fuzzy Sets and Systems, vol. 255, pp. 115-127, 2014.

[10] F. J. Cabrerizo, I. J. Pérez, W. Pedrycz, and E. Herrera-Viedma, "An improvement of multiplicative consistency of reciprocal preference relations: A framework of granular computing," in Proceedings of the 2017 IEEE International Conference on Systems, Man, and Cybernetics, (Banff, Canada), pp. 1262-1267, October 2017.

[11] W. Pedrycz and M. Song, "Analytic hierarchy process (AHP) in group decision making and its optimization with an allocation of information granularity," IEEE Transactions on Fuzzy Systems, vol. 19, no. 3, pp. 527-539, 2011.

[12] J. C. Fodor and M. R. Roubens, Fuzzy Preference Modelling and Multicriteria Decision Support. Dordrecht: Springer Netherlands, 1994.

[13] Z. Ren, Z. S. Xu, and H. Wang, "Multi-criteria group decision-making based on quasi-order for dual hesitant fuzzy sets and professional degrees of decision makers," Applied Soft Computing, vol. 71, pp. 20-35, 2018.

[14] M. Spitale, F. Catania, P. Crovari, and F. Garzotto, "Multicriteria decision analysis and conversational agents for children with autism," in Proceedings of the 53rd Hawaii International Conference on System Sciences, (Maui, Hawaii, USA), pp. 1005-1014, January 2020.

[15] A. Asemi, Z. Kazempour, and H. A. Rizi, "Using LibQUAL ${ }^{+\mathrm{TM}}$ to improve services to libraries: A report on academic libraries of iran experience," The Electronic Library, vol. 28, no. 4, pp. 568-579, 2010.

[16] Y. Gao and D. Li, "A consensus model for heterogeneous multi-attribute group decision making with several attribute sets," Expert Systems with Applications, vol. 125 , pp. 69-80, 2019.

[17] Y. Liang, J. Qin, L. Martínez, and J. Liu, “A heterogeneous QUALIFLEX method with criteria interaction for multi-criteria group decision making," Information Sciences, vol. 512, pp. 1481-1502, 2020.
[18] R. R. Yager, "Weighted maximum entropy OWA aggregation with applications to decision making under risk," IEEE Transactions on Systems, Man, and Cybernetics - Part A: Systems and Humans, vol. 39, no. 3 , pp. 555-564, 2009.

[19] L. A. Zadeh, "A computational approach to fuzzy quantifiers in natural languages," Computers \& Mathematics with Applications, vol. 9, no. 1, pp. 149-184, 1983.

[20] R. R. Yager, "Quantifier guided aggregation using OWA operators," International Journal of Intelligent Systems, vol. 11, no. 1, pp. 49-73, 1996.

[21] J. Kennedy and R. C. Eberhart, "Particle swarm optimization," in Proceedings of the 1995 IEEE International Conference on Neural Networks, (Perth, Australia), pp. 1942-1948, November/December 1995.

[22] J. Kennedy, "The particle swarm: social adaptation of knowledge," in Proceedings of the 1997 IEEE International Conference on Evolutionary Computation, (Indianapolis, USA), pp. 303-308, April 1997.

[23] Y. Shi and R. C. Eberhart, "A modified particle swarm optimizer," in Proceedings of the 1998 IEEE International Conference on Evolutionary Computation, (Anchorage, USA), pp. 69-73, May 1998.

[24] D. Wang, D. Tan, and L. Liu, "Particle swarm optimization algorithm: An overview," Soft Computing, vol. 22 , no. 2 , pp. 387-408, 2018

[25] C.-L. Hwang and M.-J. Lin, Group Decision Making Under Multiple Criteria: Methods and Applications. Berlin: Springer-Verlag, 1987.

[26] S. Liu, W. Pedrycz, A. Gacek, and Y. Dai, "Development of information granules of higher type and their applications to granular models of time series," Engineering Applications of Artificial Intelligence, vol. 71, pp. 60-72, 2018 .

[27] M. Song, Y. Jing, and W. Pedrycz, "Granular neural networks: A study of optimizing allocation of information granularity in input space," Applied Soft Computing, vol. 77, pp. 67-75, 2019.

[28] E. Herrera-Viedma, F. Chiclana, F. Herrera, and S. Alonso, "Group decision-making model with incomplete fuzzy preference relations based on additive consistency," IEEE Transactions on Systems, Man, and Cybernetics - Part B: Cybernetics, vol. 37, no. 1, pp. 176-189, 2007.

[29] R. R. Yager and D. P. Filev, "Induced ordered weighted averaging operators," IEEE Transactions on Systems, Man, and Cybernetics - Part B: Cybernetics, vol. 29, no. 2, pp. 141-150, 1999.

[30] F. Chiclana, F. Herrera, and E. Herrera-Viedma, "Integrating three representation models in fuzzy multipurpose decision making based on fuzzy preference relations," Fuzzy Sets and Systems, vol. 97, no. 1, pp. 33-48, 1998.

[31] R. R. Yager, "On ordered weighted averaging aggregation operators in multicriteria decisionmaking," IEEE Transactions on Systems, Man, and Cybernetics, vol. 18, no. 1, pp. 183-190, 1988.

[32] B. Zhang, Y. C. Dong, and Y. Xu, "Multiple attribute consensus rules with minimum adjustments to support consensus reaching," Knowledge-Based Systems, vol. 67, pp. $35-48,2014$. 